\title{
Umweltinnovationen - Anreize und Hemmnisse
}

\author{
Welchen Beitrag vermögen Innovationen im Sinne der Gewinnung und Anwen- \\ dung neuen Wissens zur Lösung drängender Probleme in Wirtschaft und Gesell- \\ schaft zu leisten? Welche Anreize und Hemmnisse für Innovationen gehen von \\ den bestehenden Politikmustern aus? Erste Antworten gibt ein kürzlich abge- \\ schlossenes Verbundprojekt zu den innovativen Wirkungen umweltpolitischer \\ Instrumente.
}

$\mathrm{D}$

Von Ulrike Lehr und Klaus Löbbe er Verweis auf Innovationen zur Lösung aktueller oder zukünftiger Probleme scheint inzwischen selbst $\mathrm{zu}$ einer Innovation geworden zu sein, die mit beeindruckender Breite und Geschwindigkeit durch alle Bereiche des gesellschaftlichen, wirtschaftlichen und sozialen Lebens diffundiert: Kaum eine Bundes- oder Landesregierung, selten eine politische Partei oder Standesorganisation, die in ihren Programmen und Aufrufen darauf verzichtet, Innovationen zur Lösung verschiedenster individueller oder kollektiver Probleme zu empfehlen. Auch im wissenschaftlichen Bereich, namentlich in den Sozial-, Politik- und Wirtschaftswissenschaften, vor allem aber in der Umweltökonomie, scheint das Interesse sprunghaft gestiegen.

Ursächlich hierfür ist wohl die Erwartung, daß Innovationen eine Lösung der anstehenden Probleme zu minimalen Kosten oder gar eine doppelte oder dreifache Dividende in Form ökonomischer, ökologischer und/oder sozialer Vorteile versprechen. Allerdings ist nicht auszuschließen, daß viele Umweltinnovationen in kurz- und mittelfristiger Perspektive durch ein „doppeltes Externalitätenproblem“ gekennzeichnet sind: Da weder der Innovator noch derjenige, der in den Umweltschutz investiert, ohne weiteres die Erträge seines Handelns an sich ziehen kann, besteht die Gefahr, daß das tatsächliche Niveau der Umweltinnovationen hinter den gesamtwirtschaftlich erwünschten Aktivitäten zurückbleibt. Soweit dies der Fall ist, fält der Politik - namentlich der Umwelt- und der Forschungspolitik - die Aufgabe zu, geeignete Rahmenbedingungen und Anreizmechanismen $\mathrm{zu}$ schaffen, durch die die Bereitschaft der Wirtschaftssubjekte gestärkt wird, umweltschonende
Neuerungen zu entwickeln, anzuwenden und zu verbreiten. Die praktische Politik kommt dieser Aufgabe in allen westlichen Industrieländern durch ein weit ausdifferenziertes System allgemeiner und spezieller Regeln nach. Im vom Bundesministerium für Bildung, Wissenschaft, Forschung und Technologie initiierten Forschungsverbund Innovationen und Umweltpolitik (FIU) haben zehn Forschungseinrichtungen in insgesamt 18 Teilstudien und einer Gesamtauswertung die im Einzelfall praktizierten Maßnahmen der Forschungs- und der Umweltpolitik im Rahmen eines umfassenden Politikansatzes überprüft (1).

Es hat sich freilich gezeigt, daß mit der inflationären Verwendung eine wachsende Unschärfe des Innovationsbegriffs einherzugehen scheint. Somit scheint es unumgänglich, zunächst den Begriff der Innovation zu präzisieren, wobei die Betrachtung a priori auf die sog. Umweltinnovationen fokussiert wird (siehe Kasten). Die Studie greift aus der Gesamtmenge der Innovationen also jene Neuerungen heraus, die zu einer Verminderung anthropogen bestimmter Übernutzungen der Umwelt führen; sie werden - der Kürze halber, aber semantisch nicht ganz zutreffend nachfolgend Umweltinnovationen genannt (2). Als solche Neuerungen gelten die Gewinnung neuen Wissens, die Anwendung neuer Erkenntnisse sowie die Änderung individueller und kollektiver Verhaltensmuster.

Der Begriff der Umweltinnovationen ist damit final definiert; er umfaßt alle Innovationen, die der Verbesserung der Umwelt dienen, gleichgültig, ob diese Innovationen auch unter anderen namentlich ökonomischen - Gesichtspunkten vorteilhaft wären. Hierzu wird im folgenden der gedanklichen Zerlegung einer Innovation in die
Umweltinnovationen umfassen

1) technisch-ökonomische Innovationen in Form

- der Entwicklung und Vermarktung neuer Produkte und der Erschließ3ung neuer Ressourcen und Inputbestände (produktbezogene Innovationen bzw. Extraktionsinnovationen),

- der Entwicklung und Anwendung never Produktionsverfahren bei der Herstellung von Gütern und Dienstleistungen und bei der Nutzung natürlicher Ressourcen (Prozeß-bzw. Ressourceninnovationen) und/oder

- der Veränderungen in der betrieblichen Organisationsstruktur, in den Unternehmensstrategien und in der Unternehmenskultur (organisationsbezogene oder strategische Innovationen);

2) institutionelle Innovationen in Form der Neugestaltung der gesamtgesellschaftlichen Rahmenbedingungen und Ordnungsprinzipien und

3) soziale Innovationen, die mit der Herausbildung (neuer) entscheidungsprägender Normen bzw. Verhaltensweisen verbunden sind und sich auch in der praktischen Anwendung neuer Lebensstile cusdrücken (3),

soweit diese die anthropogen bestimmten Umweltnutzungen vermindern, indem sie $z . B$.

zur Einsparung von Energie und Rohstoffen,

zur Reduzierung des Flächenverbrauchs und

zur Verminderung von Emissionen und Abföllen

beitrogen oder

ein gegebenes Landschaftsbild und eine gegebene Artenvielfalt erhalten.

Phasen der Entstehung, Adaption und der Diffusion gefolgt. Von hier aus werden Anreize und Hemmnisse entlang der Wirkungskette von der Ebene der Akteure über die umweltpolitische Regelung bis hin zu den allgemeinen Rahmenbedingungen analysiert.

\section{Umweltinnovationen: Entstehung...}

Die Entstehung von Umweltinnovationen wird von den Anreizen auf Akteursebene dominiert. Dies uiberrascht wenig, ist doch gerade die Forschungs- und Entwicklungstätigkeit stark mit der Kreativität und Findigkeit der Pioniere verknüpft. Nichtsdestotrotz lassen sich einige erfolgversprechende Faktoren und Muster aus den vorgefundenen Ergebnissen isolieren:

- Umweltinnovationen unterliegen weder einzelnen Determinanten noch einzelnen umweltpolitischen Instrumenten. Diese These, die sich auch als Multi-Impuls These bezeichnen läßt, kann durchgehend bestätigt werden. Allerdings hat sich auch erwiesen, daß die Impulse je 
nach Sektor oder Branche durchaus unterschiedlich sein können. Es läßt sich keine Impuls-Kombination isolieren, die zu überwiegend positiven Resultaten führt. Somit lautet die Konsequenz aus den untersuchten Fällen bestehender Regulierungsmuster und Anreizsysteme, daß „anspruchsvolle Umweltinnovationsziele politisch nur durch eine akteursspezifisch differenzierte und auch an Einzelhemmnissen ansetzende Multi-Impuls-Strategie erreichbar (sind)“(4). Besonders das Wechselspiel zwischen freiwilligen Vereinbarungen und umweltpolitischen Maßnahmen als Droh- und Kontrollmechanismus macht die Abhängigkeit innovativer Ergebnisse von Regulierungsmustern deutlich. Ebenso sind die Aspekte der Motivation einzelner Akteure vor dem Hintergrund der bestehenden Regulierung zu verstehen.

- Die Entstehung integrierter Lösungen wird durch die Nachfrage mitbestimmt. Es bestätigte sich in mehreren Fallstudien, daß die Unternehmen eine sich aufgrund von staatlichen Regelungen ändernde Nachfrage in ihre Innovationsstrategie einbeziehen. Die Produktionsentwicklung der Windkraftanlagen beispielsweise deutet auf eine ,demand pull“-Funktion hin, die durch die staatliche Betreiberförderung angeregt bzw. unterstiitzt wurde und auf die Hersteller zurückwirkte. Die dadurch ausgelösten Umweltinnovationen sind typischerweise den inkrementellen Innovationen zuzurechnen.

- Das Ordnungsrecht entfaltet seine wesentliche Wirkung über Ankündigungseffekte. In der Studie wurden Hinweise darauf gefunden, daß die Ankündigungseffekte fast stärkere Wirkung zeigen als die Implementation. Allerdings wird dieser Effekt zunichte gemacht, wenn eine Implementation nicht erfolgt; in diesem Fall werden Innovationsanstrengungen zurückgefahren.

- Das Innovationspotential weicher Instrumente hängt stark von der betriebswirtschaftlichen Rentabilität und vom Ausmaß der vorbandenen No-regret-Potentiale $a b$. Diese These konnte in einigen Fallstudien bestätigt werden. Jedoch ist auch die Wirksamkeit anderer Instrumente vom Ausmaß der No-regretPotentiale abhängig. Dies trifft in umso stärkerem Ausmaß auf die Diffusion von Umweltinnovationen $\mathrm{zu}$.

- Die Innovationswirkung des Ordnungsrechts wird durch die Trägheit der politischen Prozesse begrenzt. In den untersuchten Beispielen ist dies recht häufig der Fall. Die politi- sche Willensbildung im Vorfeld von Verordnungen ist häufig stark vom Interessenausgleich der betroffenen Unternehmen geprägt.

- Das bestehende Ordnungsrecht führt zu einer Begünstigung nachgeschalteter Technologien. Dies gilt jedoch in der Inventionsphase nicht, da die überwiegende Zahl der Studien gezeigt hat, daß das Ordnungsrecht wenig Einfluß auf die Invention hat. Somit ist die Entstehung nachgeschalteter Technologien stärker dem Beharren auf technologischen Trajektorien und unsicheren Marktsignalen zuzuschreiben.

\section{-... Adaption ...}

Die Adaptionsphase ist stärker von Instrumenten beeinflußbar als die Invention. Dabei wirken sich Abgaben und Ordnungsrecht überwiegend in den Bereichen aus, in denen der Übergang zur Diffusion (vgl. weiter unten) fließend ist. Die eigentliche Adaption, als erste - pionierhafte Anwendung, ist mehr von intrinsischer Motivation oder von Subventionen beeinflußt.

- Die Adaption von Umweltinnovationen wird durch mangelnden Zugang zu technischem Wissen gehemmt. In der Tat haben sich der Patentschutz, fehlende Marktanreize und unzureichende oder unklare rechtliche Vorgaben als Hemmnis erwiesen.

- Die Programme zur Innovationsförderung sind nicht hinreichend mit Programmen zur Adaption verzahnt. Oftmals ist eher ein zeitliches Nacheinander dieser Programme zu beobachten. Allerdings wurde dieser Umstand in den Fallstudien nicht als ein gravierendes Hemmnis gekennzeichnet.

\section{.... und Diffusion}

Insgesamt hat sich gezeigt, daß die Diffusionsphase in vergleichsweise starkem Maße durch umweltpolitische Regulierungen beeinflußt wird - und zwar auch dann, wenn andere Instrumente (etwa Steuern und Abgaben) ergänzend eingesetzt werden. Während die Inventionsförderung - sei es durch Forschungsförderung, Netzwerkbildung oder das Setzen von Standards für die Zukunft (etwa für emissionsfreie Fahrzeuge) - immer zukunftsoffen und teilweise ergebnisoffen ist, kann die Diffusionsförderung versuchen, vorhandene Technologien gezielt $\mathrm{zu}$ verbreiten. So stellen auch eine Reihe von Teilstudien eine dominante Rolle des Staates in der Diffusionsphase fest. Die untersuchten Instrumente umfassen verschiedene Abstufungen von staatlicher Intervention.
Die einfachste Diffusionshilfe ist das Gebot. Diese Feststellung gilt allerdings nur auf den ersten Blick, denn Gebote sind - außer zur akuten Gefahrenabwehr - außerordentlich schwer durchsetzbar. Beispiele gibt es u.a. aus der amerikanischen Umweltpolitik (FCKW, Katalysator). In den USA wurde mit dem Clean Air Act ein „technology forcing“-Standard festgelegt, also ein nicht an der bestehenden Technik orientierter, sondern über den Stand der Technik hinausgehender Grenzwert. Allerdings wurde seitens der Industrie der Vollzug immer wieder verzögert. Insgesamt scheint der FCKW-Ausstiegsprozeß ein anschauliches Beispiel dafür zu sein, daß gerade größere Unternehmen bereits in der Erwartung zukünftiger regulierender Maßnahmen handeln - seien sie national oder international, ordnungsrechtlich oder ökonomisch, beispielsweise um einen Wettbewerbsvorsprung gegenüber der Konkurrenz oder ,first mover"Gewinne erzielen zu können.

Für freiwillige Selbstverpflichtungen bestätigt sich die Annahme, daß die Industrie zunächst nur Reduktionsmaßnahmen anbieten wird, die bestenfalls innerhalb des No-regret-Potentials liegen (5). Politischer, gesellschaftlicher oder auch Konkurrenzdruck kann Unternehmen jedoch dazu bewegen, sich in freiwilligen Selbstverpflichtungen auf weitergehende Zusagen einzulassen. Gerade in solchen Fällen kann eine Risikominimierungsstrategie die eigentliche Motivation für die freiwillige Selbstverpflichtung sein; gemeinsames Vorgehen in bezug auf technische Standards vermindert für ein einzelnes Unternehmen die Risiken.

Zusammenfassend lassen sich die folgenden Hypothesen über Anreize und Hemmnisse zur Diffusion bestätigen :

- Das Ordnungsrecht begünstigt eine rasche Diffusion von Neuerungen. Diese These läßt sich im Lichte etlicher Ergebnisse bestätigen. Im Bereich der Diffusion lassen sich Ankündigungseffekte beobachten, die noch vor Inkrafttreten einer neuen Verordnung oder der Verschärfung einer bestehenden Verordnung auftreten.

- Die Diffusion neuer Techniken wird durch staatliche Zielvorgaben mit einer zeitlich gestuften flexiblen Instrumentierung erreicht („Threat and Control“). Die Berücksichtigung von Anpassungszeiträumen und flexibler Instrumentierung hat sich besonders in Bereichen, die mit hohen Investitionen verbunden sind, als wichtig für eine politische Durchsetzbarkeit erwiesen. 
Private Haushalte sind mit spezifischen Hemmnissen (Allokationseffekte, rechtlicher Rabmen etc.) konfrontiert, die die Technologiewahl einschränken. Diese These trifft gerade in Deutschland in erheblichem Umfang zu. Insbesondere im Bereich von Mietwohnungen ist die Technologiewahl eingeschränkt. Eine Kombination aus monetären Anreizen und Informationsangeboten hat sich bei den privaten Haushalten bewährt (6).

- Die Einführung integrierter Umwelttechniken setzt im allgemeinen eine umfassende Neuordnung der betrieblichen Produktionsabläufe voraus. Hierzu liefern einige Teilstudien anschauliche Beispiele (7).

Insgesamt zeigen die hier herausgegriffenen Ergebnisse der Teilstudien und der Gesamtauswertung in der Tat, daß Innovationen einen Beitrag zu Umweltschutz und Nachhaltigkeit leisten und in vielen Fällen den Konflikt zwischen Ökologie und Ökonomie mildern können.

\section{Anmerkungen}

(1) Klemmer, P./ U. Lehr/ K. Löbbe: Umweltinnovationen Anreize und Hemmnisse, Innovative Wirkungen umweltpolitischer Instrumente, Bd. 2, Analytica, Berlin 1999, und Klemmer, P. (Hrsg.): Umwelt und Innovation, Fallstudien zum Anpassungsverhalten in Wirtschaft und Gesellschaft, Innovative Wirkungen umweltpolitischer Instrumente, Bd. 3, Analytica, Berlin 1999.

(2) Letztlich soll nicht die Umwelt erneuert werden, wie es vielleicht die Nähe zu anderen Begriffen (Produktinnovation im Sinne eines neuen Produktes etc.) nahelegt.

(3) Die Definition technisch-ökonomischer Innovationen sowie die im folgenden getroffene Phasenunterscheidung lehnt sich Schumpeter an;

vgl. hierzu Schumpeter, J.A.: Theorie der wirtschaftlichen Entwicklung. 5. Aufl. Berlin 1952 (im Original 1926) und Schumpeter, J.A.: Business Cycles: A Theoretical, Historical and Statistical Analysis of the Capitalist Process. New York 1939. Mit der Einbeziehung institutioneller und sozialer Innovationen wird der Innovationsbegriff jedoch umfassender definiert als in der Innovationsforschung üblich.

(4) Linscheidt, B./ O. Tidelski: Innovationseffekte von kommunalen Abfallgebühren. In: Klemmer, P. (Hrsg.) a.a.0.
(5) Rennings, K. et al.: Ein Ordnungsrahmen für eine Politik der Nachhaltigkeit. In: L. Gerken (Hrsg.): Ordnungspolitische Grundfragen einer Politik der Nachhaltigkeit. Nomos, Baden-Baden 1996, S. 229-280.

(6) Lehr, U.: Innovative Wirkungen umweltpolitischer Instrumente - das Beispiel der privaten Haushalte. In: Klemmer, P. (Hrsg.) a.a.0.

(7) Vgl. z.B. Hafkesbrink, J./ M. Halstrick-Schwenk/ K. Löbbe: Abschätzung der innovativen Wirkungen umweltpolitischer Instrumente in den Stoffströmen Elektroaltgeräte/Elektronikschrott. In: Klemmer, P. (Hrsg.) a.a.0.

\section{Die Autorlnnen}

Ulrike Lehr ist wissenschafliche Mitarbeiterin der Forschungsgruppe „Energiewirtschaft", Klaus Löbbe ist Leiter der Forschungsgruppe „Sektorale Strukturanalysen", beide am Rheinisch-Westfälischen InstituI für Wirtschaltsforschung (RWI).

Kontakt: RWI, Hohenzollernstr. 4-5, 45134 Essen, Tel. 0201/8149-237, Fox -200,

E-mail: lehr@rwi-essen.de

\section{Innovationspotentiale von Umwelftechnologien}

In den FIU-Studien wird das Innovationsgeschehen durch die "Instrumentenbrille" betrachtet. Im gleichen Zeitraum hat das Umwelthundesamt eine Studie in Auftrag gegeben, die Innovationspotentiale stärker durch die Brille des Technikers analysiert (1). Die Ergebnisse werden im folgenden kurz dargestellt und kommentiert.

In der Studie werden die Entwicklungsmöglichkeiten von 15 ausgewählten Umwelttechniken analysiert, ihr Beitrag zur Umweltentlastung ermittelt und ihre Verbreitung untersucht. Es handelt sich also um ex-post Analysen, die auf spezielle Techniken fokussiert sind und deren Ergebnisse dann verallgemeinert werden. Die Teilstudien beeindrucken durch technische Kompetenz, dos Verfahren hat allerdings ouch den Nachteil, daß das Innovationssystem einer (nationalen) Branche nur am Rande aufleuchtet. Und das dürfte ganz maßgeblich das Innovationsgeschehen prägen. Umwelttechnologien werden hier ols „Querschnittstechnologien" betrachtet, also im wesentichen als Rekombinofionen von bereits vorhandenen technischen Potentialen. Das ist ex-post für die betrachteten Umwelttechnologien wohl richtig, könnte aber dos Ausmaß der erforderlichen Innovationstätigkeit verfehlen (vgl. den Beitrag von Hübner in diesem Heff). Damit muB hinter die Übertragbarkeit dieser ex-post Betrachtung auf ex ante Überlegungen zumindest ein Fragezeichen gesetzt werden

Die wesentlichen Determinanten des Innovationsgeschehens werden wie folgt angegeben:

. .Politik und Gesetzgebung,
- das Rentabilitötskalkül der Unternehmen,

- die outonome technische Entwicklung und

das Umweltbewußtsein in der Gesellschaft" (S. 353).

Als dominante Hemmnisse werden genannt:

- "Mongelnde wirtschaftliche Attraktivitöt der Technik (Rentabilitätrsdefizit),

- Anwendungsgrenzen durch unzureichende technische Reile der Technik (Technologiedefizit) und

- Informationsmängel über die Technik (Informationsde(izit)" (S. 356).

Daneben nehmen auch rechtliche Defizite eine herousgehobene Position ein. Diese Aufzählungen sind wenig überroschend und relativ allgemein.

\section{Interessante Ergebnisse}

Interessant wird die Studie, wenn sie etwas detaillierter wird. So wird beispielsweise die Bedeutung der Bildung von strategischen Allianzen, „.... welche die auf verschiedenen Stufen der Wertschöpfungskette vorhandenen Kompetenzen bindeln..." mit Blick ouf die internationale Wettbewerbsposition hervorgehoben. Durchous ouch heute noch zutreffend wird weiter formuliert: „Die Bedeutung solcher Allianzen und von Gemeinschaftsunternehmen dürfte in Deutschlond noch verkannt werden. Auch die Einbindung sozialwissenschaftlicher Disziplinen (einschlieflich der Managementforschung) zur Beherrschung der Kooperationsprobleme, die ouftreten können, besitzı keine Tradilion" (S. 360).

Eine ondere Einsicht mit weitreichenden Folgen wird, ähnlich wie in der bei Lehr/ Löbbe zitierten FIU-Studie zur
Elektronikschrottverordnung, wie folgt ausgedrückt: ${ }_{E}$ Es ist fraglich, ob die Umweltpolitik sich der Aufmerksamkeit, die ihrem Handeln, aber auch ihrem Zögern von seiten der Wirtschaftsakteure zuteil wird, bewuBt ist ${ }^{4}$ (S. 362). Wer denkt bei dem Stichwort "zögern" nicht an die Umsetzung des Kreisloufwirtschaftsgesetzes. Weiter heißt es mit Blick ouf das Innovationsgeschehen: „Schlingerkurse erzeugen demgegenüber in der Wirtschaft eine vorsichtige, abwortende Haltung" (S. 353).

Und es findet sich ouch schon in dieser Studie die Empfehlung, zur Verbesserung der ökologischen Richtungs- und Geschwindigkeitssicherheit von Umweltinnovationen ein "zukunftssicheres Umweltprogramm" zu entwickeln, das die wesentlichen ${ }_{n}$..Problemfelder und Handlungserfordernisse definiert, Ziele setzi und Realisierungszeilröume autzeigt..." (S. 365); ein Gedanke der sich hinter Jänickes Idee vom nationalen Umweltplan verbirgt (2). Daß zu diesem Zweck Konsensbildung mit den relevanten Wirtschaftsakteuren erforderlich ist, dürfte fast schon selbstevident sein.

\section{Stefan Zundel}

\section{Anmerkungen}

(1) Umweltbundesamt (Hisg.): Innovationspotentiale von Umweltrechnologien - Innovationsstrategien im Spannungsfeld von Technologie, Ökonomie und Ökologie. Heidelberg 1998.

(2) Vgl. z.B. Jünicke, M. et al: Konsensuale Zielbildung. Nationole Umweltplanung als never Ansatz der Umweltpolitik. Ökologisches Wirtschaften 2/97, S. 6-7. 
(c) 20I0 Authors; licensee IÖW and oekom verlag. This is an article distributed under the terms of the Creative Commons Attribution Non-Commercial No Derivates License (http://creativecommons.org/licenses/by-nc-nd/3.o/), which permits unrestricted use, distribution, and reproduction in any medium, provided the original work is properly cited. 